\title{
Magnetic structure of the solar transition region as observed in various ultraviolet lines emitted at different temperatures
}

\author{
E. Marsch ${ }^{1}$, G.-Q. Zhou ${ }^{2}$, J.-S. He ${ }^{2}$, and C.-Y. Tu ${ }^{1,2}$ \\ 1 Max-Planck-Institut für Sonnensystemforschung, Katlenburg-Lindau, Germany \\ e-mail: marsch@mps.mpg.de \\ 2 Department of Geophysics, Peking University, Beijing, PR China \\ e-mail: cytu@public3.bta.net.cn
}

Received 23 May 2006 / Accepted 28 June 2006

ABSTRACT

\begin{abstract}
Aims. The structure of the solar transition region (TR) in a polar coronal hole of the Sun is studied. In particular, the detailed association of the coronal magnetic field (carpet) with the radiance patterns of the TR, when seen in various far ultraviolet (FUV) emission lines, is investigated.

Methods. A detailed comparison is made of the coronal magnetic field, as obtained by extrapolation of the NSO/Kitt-Peak photospheric field to heights of several tens of megameters, with the radiances of many FUV lines, which are emitted by ions of various elements at different ionization stages, corresponding to different local coronal temperatures. By a correlation analysis of the emission pattern with the magnetic field (network and carpet of loops), the so-called correlation height of the emission can be determined. By its help and through a correlation analysis the magnetic nature of the emission regions and the temperature structure of the TR can be better revealed and understood.

Results. In particular, at mesoscopic scales of several megameters the regions with strong emission (originating from multiple small closed loops) are found to be located at low heights, whereas weak emissions (coming from locally open, i.e. far reaching fields) appear to originate at greater heights. These findings are consistent with similar results obtained at large scales for large-size loops and big coronal holes.

Conclusions. Our correlation-height analysis of the emission lines confirms the notion that plasma at different temperature can coexist at the same height. The TR is not thermally stratified but strongly nonuniform and magnetically structured.
\end{abstract}

Key words. Sun: corona - Sun: atmosphere - Sun: magnetic fields - Sun: transition region - Sun: UV radiation - Sun: photosphere

\section{Introduction}

The solar atmosphere is known to be structured on all scales by the Sun's magnetic field, which controls the mass supply to the solar corona and the energy and momentum transport of the coronal plasma. Our knowledge of the magnetic field in the transition region (TR) and corona mainly stems from extrapolations of the field as measured in the photosphere and chromosphere. The modern techniques to construct the force-free coronal magnetic field from photospheric magnetograms were for example reviewed by Wiegelmann \& Neukirch (2002).

The magnetograms may either be obtained from space, like with MDI (Michelson Doppler Imager, Scherrer et al. 1995) on SOHO (Solar and Heliospheric Observatory), or the ground, e.g. from the Spectromagnetograph at National Solar Observatory/Kitt Peak (NSO/KP), Harvey \& Harvey (1976), which we used here. The consideration of the simultaneous magnetic field data enables us to carry out a deeper and more complete analysis of the SOHO ultraviolet images and Doppler maps of the TR and corona. By imaging in the far and extreme ultraviolet (FUV, EUV) light one can render the coronal plasma visible, and through spectroscopy diagnose the plasma state by means of the many emission lines that originate from a multiplicity of heavy ions confined in the coronal magnetic field. For a comprehensive recent review of this field see Wilhelm et al. (2004). Certainly, the magnetic field is indispensable if one wants to fully interpret and better understand the ultraviolet emission patterns (in terms of line radiances, Doppler shifts, and widths).

The Extreme Ultraviolet Imaging Telescope (EIT, Moses et al. 1997) and the Solar Ultraviolet Measurements of Emitted Radiation (SUMER, Wilhelm et al. 1997; Lemaire et al. 1997) spectrometer on the Solar and Heliospheric Observatory (SOHO) mission and the imager on the Transition Region and Coronal Explorer (TRACE, see e.g. Aschwanden et al. 2002) have in the past decade provided unprecedented views and diagnostics of open and closed coronal structures, such as funnels, holes, streamers, prominences, loops and active regions (ARs), and have unravelled their complex magnetic fields through imaging of the spatial distribution of the tenuous plasma confined therein. For a review of this dynamic and intricate corona see Aschwanden et al. (2001).

It is exactly this combination of field and plasma data which we will use here in a comprehensive way to study the TR and magnetic carpet in a southern polar coronal hole. The new aspect of our analysis is the correlative use of measurements, stemming from SUMER, MDI and NSO/KP, together with the magnetic field obtained by force-free extrapolation after the theory of Seehafer (1978). Similar analyses have already been carried out by Marsch et al. (2004) to study the plasma flows in ARs, by Xia et al. (2003) and Wiegelmann et al. (2005) to link flows and fields in an equatorial coronal hole $(\mathrm{CH})$, by Tu et al. (2005a) to study the solar wind origin in a coronal funnel, and by 


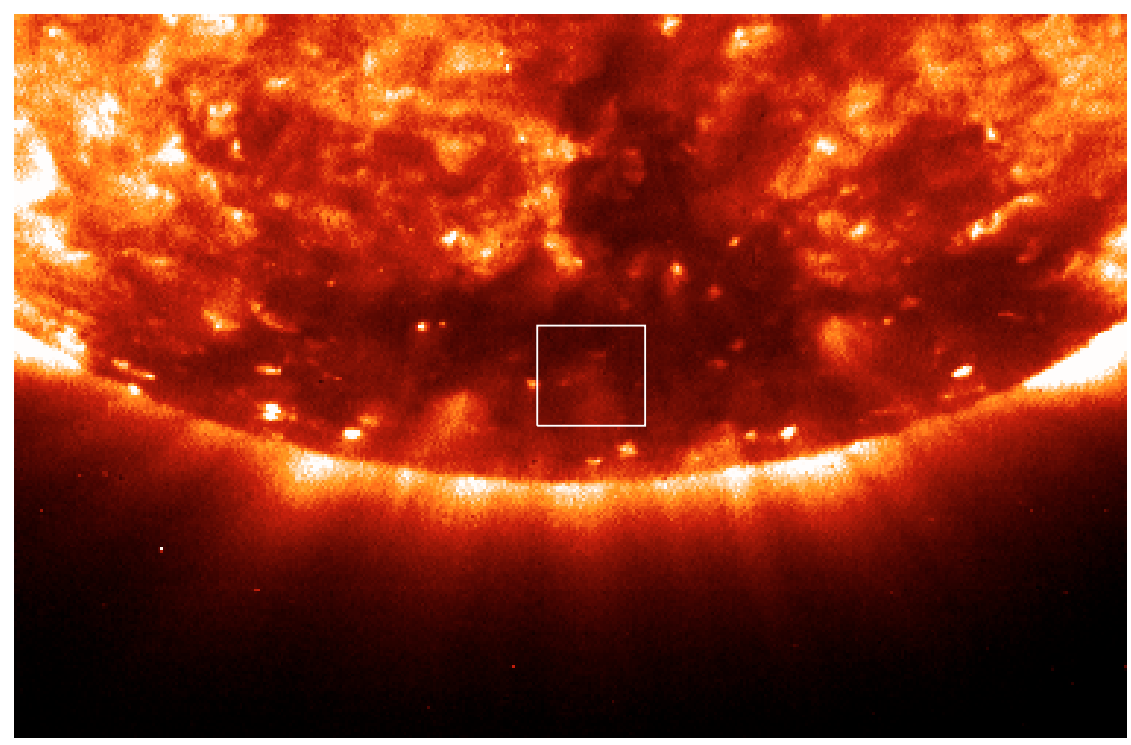

Fig. 1. EIT image, taken on 16 April 1996 at 16:18:10 UT in the Fe $17.1 \mathrm{~nm}$ line, of a selected area of the south polar coronal hole, in which the SUMER instrument took raster images (indicated by the white box) in various FUV emission lines.
Tu et al. (2005b) to determine the FUV emission height in a quiet-Sun region. Here we will not describe the field extrapolation technique, since it was explained and derived at length in the papers cited and references therein.

Tu et al. (2005a,b) introduced the concept of the correlation height of the source of a FUV emission line. For this purpose, the measured photosphere magnetic field was first extrapolated, as a force-free field by using the formula of Seehafer (1978), in a cubic volume to a height of $40 \mathrm{Mm}$. Then they calculated at different heights the correlation coefficient between the 2D distribution of any observed emission parameter, such as the line intensity or the Doppler shift, and the $2 \mathrm{D}$ distribution of the vertical component of the extrapolated magnetic field. The height at which the correlation coefficient had its maximum was called correlation height of the emission. It may be considered as a rough estimate of the real height of the radiation source. At the true emission height, the (horizontal 2D) density distribution and vertical motion of the emitting ions are largely controlled by the vertical magnetic field component. So they both should be correlated with each other, and the height of their maximal correlation should approximately represent the real height of a radiation source. If this is more widely spread in altitude, the correlation height may be considered some kind of average emission height.

The correlation between the square root of an emission-line intensity (as a proxy for the electron density) and the vertical component of the magnetic field may be, as suggested by $\mathrm{Tu}$ et al. (2005c), understood as the consequence of magnetoconvection, with the field being frozen into the flow. A possible correlation between magnetic field and plasma density may also be expected from the theoretical point of view that the heating flux density (being proportional to the density) entering loops is expected to scale with some power of the field strength, and according to some recent work by Schrijver et al. (2004) should be proportional to the base field strength.

The main goal here is to analyse coronal hole plasma radiance pattern in many different TR lines in association with the magnetic field. In particular, two selected regions in a polar $\mathrm{CH}$ will be studied, and the related magnetic field topologies and coronal plasma temperatures will be scrutinized. Many spectroscopic observations that were made in the past years with $\mathrm{SOHO}$ and TRACE revealed direct evidence for sizable plasma velocities and the multiple-temperature structure of AR loops (for
Table 1. The solar coordinates in $x$ and $y$ for the edges of the maps of the 12 emission lines considered.

\begin{tabular}{ccccccc}
\hline \hline No. & Line & $\lambda / \mathrm{nm}$ & $x_{\min } /^{\prime \prime}$ & $x_{\max } /^{\prime \prime}$ & $y_{\min } /^{\prime \prime}$ & $y_{\max } /^{\prime \prime}$ \\
\hline 1 & Si I & 125.89 & -32.27 & 73.63 & -919.80 & -829.05 \\
2 & N II & 108.57 & -32.17 & 69.06 & -922.75 & -827.15 \\
3 & S II & 125.95 & -32.27 & 73.63 & -920.80 & -829.05 \\
4 & Si II & 126.50 & -32.30 & 73.60 & -919.80 & -830.10 \\
5 & F III & 112.49 & -32.19 & 71.31 & -920.78 & -828.10 \\
6 & He II & 108.49 & -32.17 & 69.06 & -922.75 & -827.15 \\
7 & C II & 133.57 & -32.24 & 75.88 & -920.82 & -827.99 \\
8 & C III & 97.70 & -32.25 & 64.50 & -920.70 & -828.25 \\
9 & Si IV & 139.38 & -32.18 & 78.13 & -921.84 & -827.94 \\
10 & O III & 83.37 & -32.32 & 57.69 & -921.60 & -828.39 \\
11 & S IV & 106.27 & -29.99 & 69.06 & -921.73 & -828.21 \\
12 & O VI & 103.76 & -32.25 & 66.81 & -921.73 & -828.20 \\
\hline
\end{tabular}

reviews of this broad subject see Harrison et al. 1997; Brekke 1998; Kjeldseth-Moe 2003).

Here we attempt to shed new light on the complex structure of the TR at the bottom of a polar $\mathrm{CH}$, by studying the radiances of various coronal emission lines measured by SUMER. Many lines were used to achieve a continuous temperature coverage. Unlike in many earlier studies, we investigate these emission patterns here in close connection with the coronal magnetic field that was constructed by a force-free extrapolation from photospheric magnetograms.

The solar image we use was observed by EIT on 16 April 1996, with the region observed by SUMER being indicated as a white rectangle. The rectangles for each of the 12 emission lines used in the subsequent analysis are not all shown in this figure, because they are very close to the special region shown in Fig. 1. In detail, the coordinates of the regions for the different emission lines are given in Table 1.

\section{Data analysis techniques and methods}

The SUMER observations analysed here were made in 1996 on 16 April from 8:21 to $15: 48$ UTC in a south polar region on the solar disk. The selected spectral window covers quite a wide spectral range, which includes the lines Si I $(125.88 \mathrm{~nm})$, Si II $(126.47 \mathrm{~nm})$, Si IV $(139.38 \mathrm{~nm})$, O III $(83.37 \mathrm{~nm})$, O VI $(103.76 \mathrm{~nm})$, C II $(133.57 \mathrm{~nm})$, C III $(97.70 \mathrm{~nm})$, 
Table 2. Twelve emission lines in the far ultraviolet, with their wavelengths and formation (electron) temperatures. In Cols. 6-13, the logarithm of the effective temperature and related thermal speed, as based on the line width, and the correlation height and correlation coefficient are listed, for sub-region 1 and subsequently sub-region 2 .

\begin{tabular}{|c|c|c|c|c|c|c|c|c|c|c|c|c|}
\hline No. & Line & $\lambda / \mathrm{nm}$ & $\log (T / \mathrm{K})$ & $T / \mathrm{K}$ & $\log \left(T_{1} / \mathrm{K}\right)$ & $V_{1} / \mathrm{km} \mathrm{s}^{-1}$ & $h_{1} / \mathrm{Mm}$ & $c_{1}$ & $\log \left(T_{2} / \mathrm{K}\right)$ & $V_{2} / \mathrm{km} \mathrm{s}^{-1}$ & $h_{2} / \mathrm{Mm}$ & $c_{2}$ \\
\hline 1 & Si I & 125.878 & 3.87 & 7410 & $5.31 \pm 0.16$ & $11.05 \pm 2.14$ & 10.0 & 0.11 & $5.33 \pm 0.16$ & $11.33 \pm 2.11$ & 1.0 & 0.51 \\
\hline 2 & $\mathrm{~N}$ II & 108.571 & 4.4 & 15100 & $5.39 \pm 0.16$ & $17.26 \pm 3.01$ & 9.0 & 0.40 & $5.54 \pm 0.10$ & $20.32 \pm 2.29$ & 2.5 & 0.50 \\
\hline 3 & S II* & 125.953 & 4.2 & 15800 & $5.70 \pm 0.13$ & $16.19 \pm 2.37$ & 11.5 & 0.60 & $5.75 \pm 0.10$ & $17.83 \pm 2.02$ & 2.0 & 0.37 \\
\hline 4 & Si II & 126.500 & 4.26 & 18200 & $6.21 \pm 0.07$ & $30.96 \pm 2.51$ & 10.5 & 0.57 & $6.29 \pm 0.06$ & $33.80 \pm 2.28$ & 5.5 & 0.46 \\
\hline 5 & Fe III & 112.488 & 4.4 & 25100 & $5.94 \pm 0.14$ & $16.23 \pm 2.99$ & 10.0 & 0.43 & $5.90 \pm 0.14$ & $15.48 \pm 2.45$ & 3.0 & 0.52 \\
\hline 6 & He II & 108.494 & 4.7 & 50100 & $4.55 \pm 0.19$ & $12.18 \pm 2.60$ & 6.0 & 0.41 & $4.67 \pm 0.13$ & $13.96 \pm 2.08$ & 2.5 & 0.52 \\
\hline 7 & C II & 133.571 & 4.71 & 51300 & $5.45 \pm 0.13$ & $20.00 \pm 3.04$ & 5.5 & 0.35 & $5.65 \pm 0.15$ & $25.11 \pm 4.31$ & 3.0 & 0.49 \\
\hline 8 & C III & 97.703 & 4.8 & 63100 & $5.56 \pm 0.13$ & $22.52 \pm 3.32$ & 11.5 & 0.35 & $5.71 \pm 0.12$ & $26.62 \pm 3.66$ & 3.0 & 0.23 \\
\hline 9 & Si IV & 139.378 & 4.84 & 69100 & $5.66 \pm 0.14$ & $16.56 \pm 2.65$ & 40.0 & 0.42 & $5.65 \pm 0.13$ & $16.35 \pm 2.58$ & 2.0 & 0.46 \\
\hline 10 & O III & 83.374 & 4.9 & 79400 & $5.47 \pm 0.18$ & $17.48 \pm 3.09$ & 10.5 & 0.35 & $5.48 \pm 0.10$ & $17.70 \pm 2.07$ & 5.5 & 0.41 \\
\hline 11 & S IV & 106.266 & 5.31 & 204000 & $5.74 \pm 0.23$ & $17.02 \pm 5.42$ & 30.0 & 0.44 & $5.65 \pm 0.21$ & $15.33 \pm 4.34$ & 2.0 & 0.45 \\
\hline 12 & O VI & 103.764 & 5.47 & 295000 & $5.53 \pm 0.10$ & $18.89 \pm 2.26$ & 11.0 & 0.40 & $5.52 \pm 0.08$ & $18.46 \pm 1.74$ & 2.5 & 0.49 \\
\hline
\end{tabular}

* Line blend with O VI $125.954 \mathrm{~nm}$, in second order.

S II $(125.95 \mathrm{~nm}), \mathrm{S}$ IV $(106.27 \mathrm{~nm}), \mathrm{N}$ II $(108.57 \mathrm{~nm})$, He II $(108.49 \mathrm{~nm})$, Fe III $(112.49 \mathrm{~nm})$. The formation temperature of these lines ranges from $7.4 \times 10^{3} \mathrm{~K}$ to $3.0 \times 10^{5} \mathrm{~K}$, covering the temperature range from the chromosphere to the transition region. The slit with angular size of $1^{\prime \prime} \times 120^{\prime \prime}$ in the north-south direction was used. The original data set included many more lines (more than 40 for a good temperature coverage), but it turned out that many of them were too low in radiance, and had to be discarded because of bad photon statistics and a correspondingly high noise level.

The selected data set was originally derived from measurements made in ten different spectral windows, yielding ten data subsets, each of which consisted of 120 exposures. The exposure time for each slit position was $20 \mathrm{~s}$. As to the size of the whole data set on the Sun, the spatial dimension in the eastwest direction ranges from $32.80^{\prime \prime}$ east to $80.44^{\prime \prime}$ west, while in the north-south direction from $923.86^{\prime \prime}$ south to $825.30^{\prime \prime}$ south. The region studied is shown in Fig. 1 by a white rectangle superimposed on the Fe IX/X image observed by EIT (ExtremeUltraviolet Imaging Telescope) at 16:18:10 on 16 April 1996. This EIT image data was obtained from the SOHO website.

To prepare the data for analysis, the standard SUMER procedures for correcting and calibrating the individual detector images were applied. The continuum radiation was subtracted before the spectra were integrated to calculate line radiances. In these calculations, the full spectral window for each line was defined to extend from the pixel with minimum counts on the left to the one with minimum counts on the right of the central line profile. The lines used in the study are strong enough to provide sufficient counts per pixel and ensure low noise level. The lines are together with other relevant parameters composed in Table 2, which shows that the selected 12 lines provide a fair temperature coverage of the solar TR and low corona. The line formation (electron) temperatures are given together with the effective ion temperature as derived from their lines widths.

In order to get information on the emission heights of these lines, we made correlation analyses between their radiance maps and the chart of the $B_{z}$ magnetic-field component as obtained by an extrapolation of the magnetogram data obtained by NSO/KP (National Solar Observatory/Kitt Peak). The magnetogram was taken by NSO/KP from 15:25:28 to 16:20:04 UTC on the same day, with spatial resolution elements of 1 ". $15 \times 11^{\prime \prime} 15$. The NSO/KP observation time was close to the period of the SUMER raster scan. We thereby assumed that both the magnetic field and the radiation pattern essentially did not change during this period.

To facilitate a comparison of the two data sets, we first transferred the positions of the radiance sources as observed by SUMER to the position where the sources, considering that they moved at the solar rotation rate, would have been in the middle of the NSO/KP observation period (15:52:46). We then selected those magnetograms corresponding to the region of the respective SUMER raster scan for any line considered. Then by means of interpolation we rescaled both, the magnetogram and the transferred SUMER image, so as to have the same spatial resolution element of $1^{\prime \prime} \times 1^{\prime \prime}$. For the magnetic field extrapolation to the corona we used the method described by Seehafer (1978), for calculating a linear force-free field from a given photospheric magnetogram and for a fixed value of the parameter $\alpha$. This method gives the components of the coronal magnetic field in terms of a Fourier series of the photospheric magnetogram, and was successfully applied before, e.g. by Marsch et al. (2004), Wiegelmann et al. (2005) and Tu et al. (2005a).

The originally measured magnetogram covers a rectangular region extending from 0 to $L_{x}$ in $x$ (east-west coordinate), and from 0 to $L_{y}$ in $y$ (north-south coordinate). Then the rectangle was artificially extended to a four times bigger region, covering the square from $-L_{x}$ to $L_{x}$ and from $-L_{y}$ to $L_{y}$. For this extension, the antisymmetric mirror images of the original magnetograms were used in the extended region, see e.g. Wiegelmann $\&$ Neukirch (2002). In our case, the radiance map of each line is spatially slightly different from that of another line, due to the way the SUMER raster scans were obtained. In our analysis we chose a different magnetogram and separate parameters $L_{x}$ and $L_{y}$ for each line, respectively. However, the differences were minor, for example, $L_{x}=91$ and $L_{y}=194$ for Si I, and $L_{x}=86$ and $L_{y}=199$ for O VI. There was almost the same angle, $65.7^{\circ}$, between the line of sight (LOS) and the radial direction for all of the lines. Geometrical distortion effect were removed by accounting for the cosine of the angle between the LOS and radial direction for each line, respectively.

The magnetogram was extracted from the NSO/Kitt Peak observational data in order to calculate the components of the magnetic field vector using the method of Seehafer (1978). The boundary conditions that are required by that method may cause some artificial effects in the solution. For reducing such effects, the region from which we selected the magnetic data for the extrapolation was defined to be larger than those regions from which we selected SUMER data (Tu et al. 2005a,b). The final 

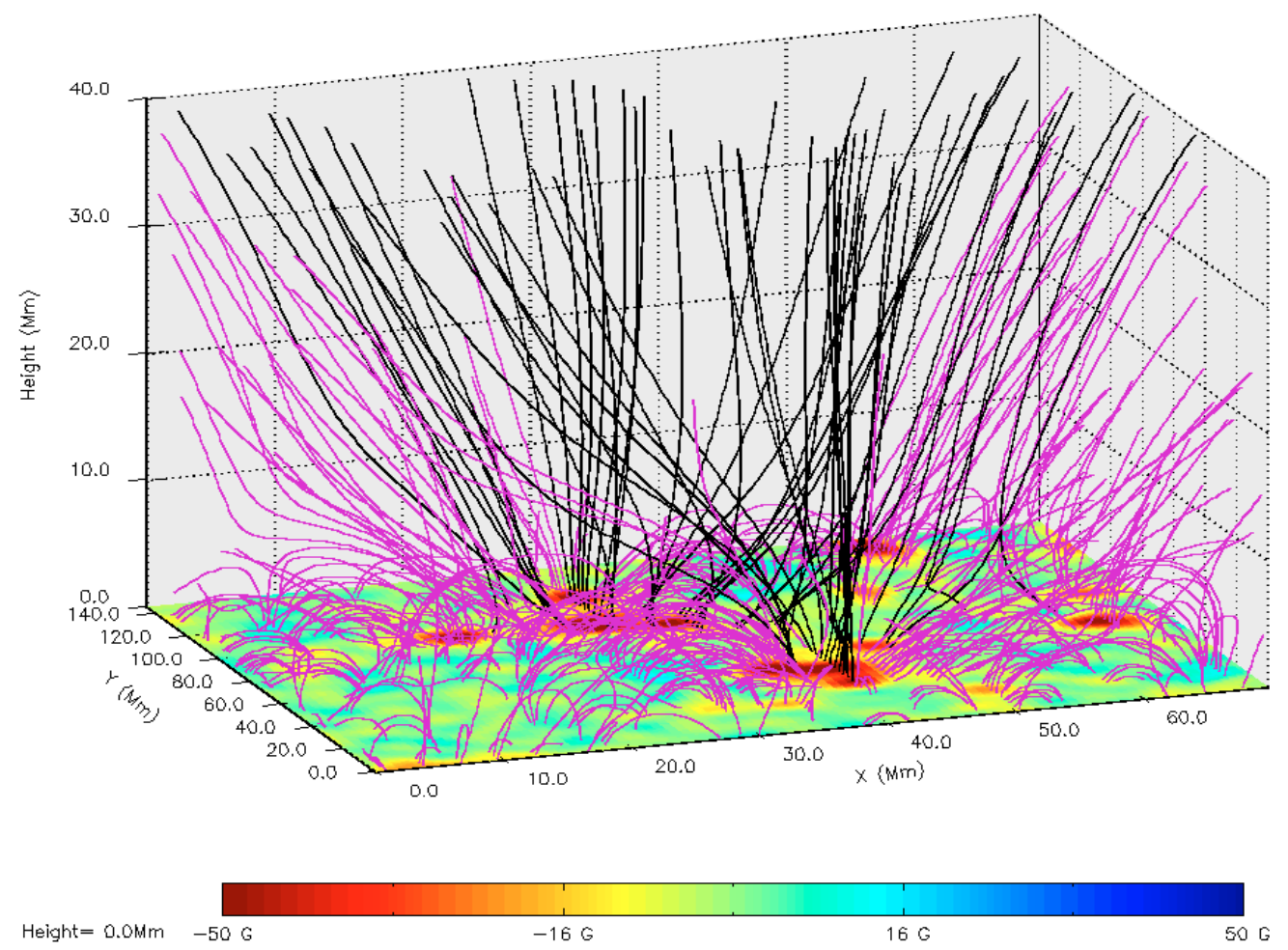

Fig. 2. Three-dimensional magnetic field in the TR and lower solar corona. The black curves illustrate open (defined as open if reaching the top of the box) and the magenta ones closed field lines, which are extrapolated from the vertical component of the magnetic field in the photosphere up to a height of $40 \mathrm{Mm}$. The bottom layer shows the colour-coded $B_{z}$ (see the colour bar below) on the photosphere. Only the strong fields with $\left|B_{z}\right|>5$ Gauss at the photosphere are displayed in the form of field lines, in order to give a clear view of the tangled loops and open field-line bundles.

magnetic region is larger than that of each respective SUMER raster scan for any considered line by $60^{\prime \prime}$ in the north, east and west direction, and by $30^{\prime \prime}$ in the south direction. By doing so, we hope to have reduced the effects of the boundaries, and to make sure the result of the extrapolation be as reliable as possible.

For the force-free parameter $\alpha$, we finally chose the value zero, corresponding to a potential field. The magnetic field was then extrapolated to a height of $40 \mathrm{Mm}$, with height intervals of $0.5 \mathrm{Mm}$. The field is shown in Fig. 2, which illustrates its topology and allows to infer where the coronal field is closed (magenta colour) and locally open to the top of the box (black colour). The magnetic carpet of closed loops is low (reaching only heights up to $10 \mathrm{Mm}$ ), and there are several regions of strong field (about 50 Gauss) concentrations which are the footpoints of the open fields and strongly expand as field-line bundles with coronal height. They merge to form the large-scale magnetic field of the $\mathrm{CH}$ shown in Fig. 1.

In the correlation analysis between the radiance of each line and the extrapolated magnetic field, we chose a photospheric magnetogram that was smaller than the transferred radiance map and confined by that map. In this way there was no need to use the extrapolated magnetic data near the boundaries. The magnetogram was then moved around on the radiance map to find the position of maximal correlation, and in order to redefine the relative solar position of the corresponding radiance map and calibrate its coordinates by means of the ones for the magnetogram. The relocated radiance map was then correlated with the extrapolated magnetic field at various given heights between 0 and $40 \mathrm{Mm}$. The height at which the maximal correlation coefficient was obtained is called correlation height after Tu et al. (2005b).
The resulting coordinates of the respective radiance maps are contained in Table 1.

\section{Results}

The area shown in Fig. 1 by the small white rectangle in the $\mathrm{CH}$ is the region of interest. It was scanned by SUMER in about 40 different VUV emission lines, out of which only the 12 lines listed in Table 2 were judged as being bright enough to be useful for our study. We present the radiance maps of eight selected lines in the subsequent Fig. 3, which shows the emission distributions of these lines that were all measured in the same $\mathrm{CH}$ region of the Sun. After having transferred the positions of the emission sources as observed by SUMER to the positions where they should be have been located at the NSO/KP observation time, we selected the region where the radiance maps well overlapped. This region extends in the $x$-coordinate from $30.0^{\prime \prime}$ east to 66.8" west, and in $y$ from $919.8^{\prime \prime}$ south to $830.1^{\prime \prime}$ south. The names and wavelengths of the eight lines are indicated on the left below the respective map. The radiance is given in arbitrary units and coded in colour as indicated by the bars.

Close inspection of the eight frames of Fig. 3 indicates that the TR in this region of the polar $\mathrm{CH}$ is structured on smaller scales. There are several bright points visible in the $\mathrm{CH}$ as illustrated in Fig. 1. Two of them fall into our scanned rectangle. They are clearly discernable and centred in the location at coordinate $x=40^{\prime \prime}$ and $y=60^{\prime \prime}$, as well as at $x=50^{\prime \prime}$ and $y=30^{\prime \prime}$. These bright regions correspond to the bundle of closed loops shown in magenta colour in the foreground of Fig. 2, with coordinates in $x$ between $20^{\prime \prime}$ and $30^{\prime \prime}$. Similarly, the oppositely located bright region relates to a bushel of closed field lines that 

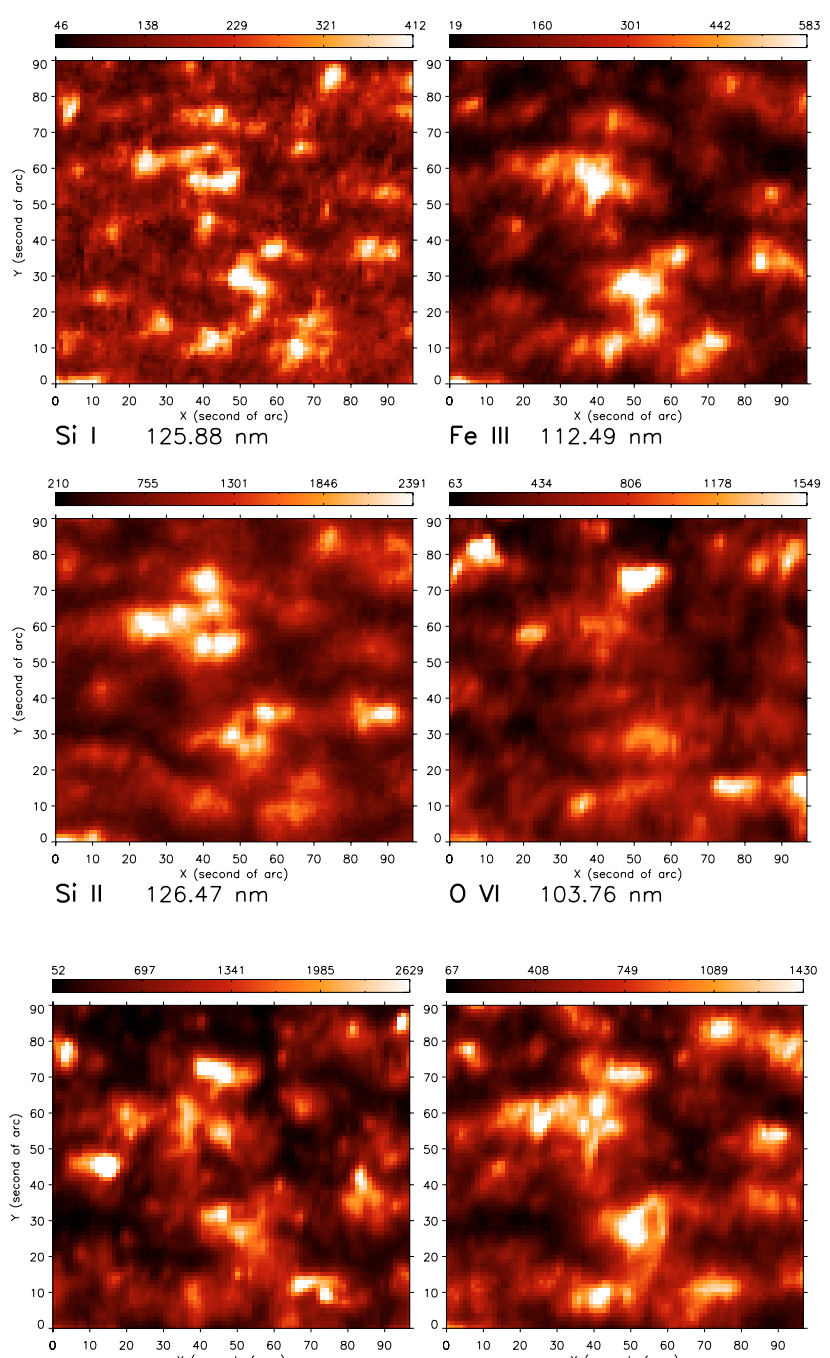

Si IV $139.38 \mathrm{~nm}$
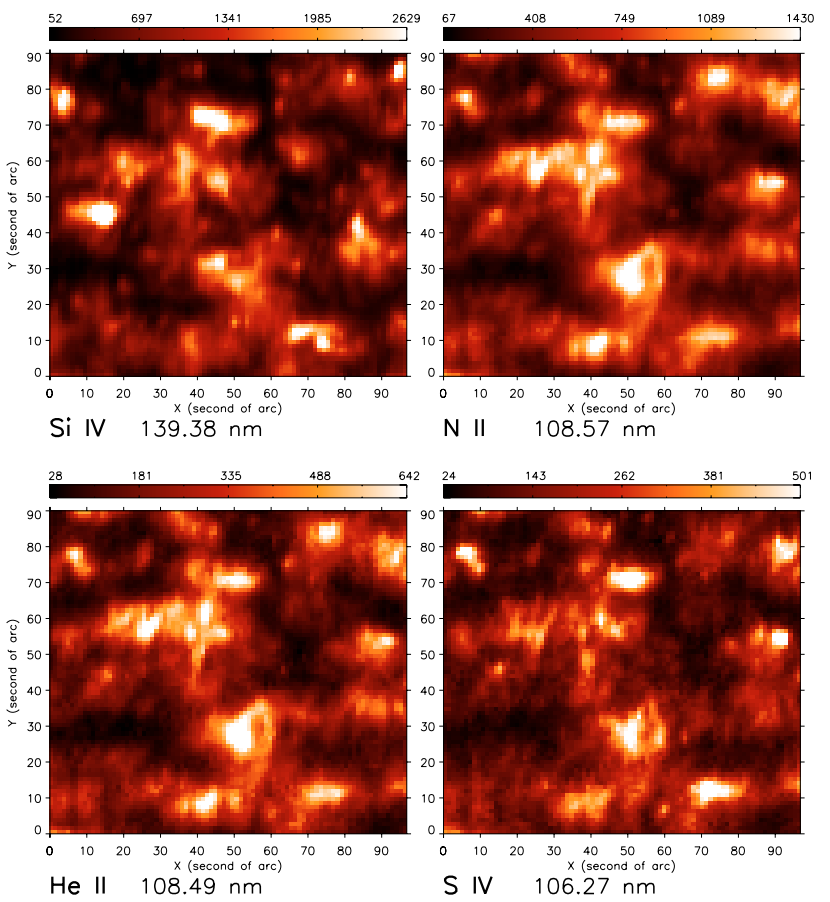

$\mathrm{N}$ II $\quad 108.57 \mathrm{~nm}$

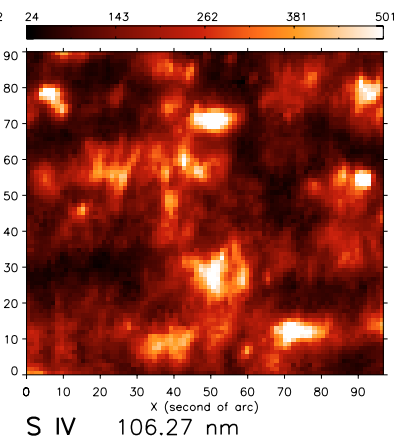

Fig. 3. The radiance distributions of eight selected emission lines measured in the same coronal hole region of the Sun. After transferring the positions of the emission sources as observed by SUMER to the positions where the emission sources should be located at the NSO/KP observation time, we selected the region where these radiance maps overlapped. The radiance, respectively, is given in arbitrary units coded in colour as indicated by the bars.

originate near an extended (over about 100") row of open (to the top of the extrapolation box) field lines, which are connected with the dark grove that is best visible in the hotter TR lines of $\mathrm{S}$ IV and O VI. Their footpoint field strength exceeds 50 Gauss.

A comparison of the eight frames of Fig. 3 indicates substantial fine structure at a few seconds of arc (several Mm) with varying brightness depending on the line temperature. In the emission in Si I at $7400 \mathrm{~K}$ there appear features characteristic of the magnetic network. The emission is generally more uniform, but also shows the two bright patches. The emission pattern in the hotter lines is more structured. In lines hotter than $200000 \mathrm{~K}$ there clearly appear, in addition to the bright regions, some dark lanes and groves which seem to relate mostly to open magnetic field lines. Yet, these features are already observable at the cooler $(25000 \mathrm{~K})$ lines of Fe III and $(50000 \mathrm{~K})$ of He II. The frames shown are representative also for the images in the other lines not shown here. Note that we cover the lower TR well in temperature (see Table 2 again) with the images used here.

When comparing Figs. 2 with 3, the shortcomings of integrated line-of-sight (LOS) observations are obvious. What in reality is a radially structured section of the solar TR and corona appears in the SUMER images only as a horizontally structured reduced image due to LOS integration effects. The $3 \mathrm{D}$ nature of the atmosphere cannot be fully reconstructed, but comparison with extrapolations like the one shown in Fig. 2 can help in identifying the 3D properties of the plasma structures underlying the ultraviolet emission. As has been demonstrated by Tu et al. (2005b), one can determine what was called the correlation height, by means of a correlation analysis of the radiance maps with the magnetic field maps at different heights. The emission is suggested to originate mostly from the layer for which the correlation coefficient is found to be maximal.

We used this technique here to determine the emission heights of all lines. Results are only shown for one cold line, Si I at $7400 \mathrm{~K}$, and one hotter TR line, O VI at about $300000 \mathrm{~K}$. In Fig. 4 the height profiles of the correlation coefficient between the observed radiance of these lines and the unsigned longitudinal magnetic field $\left|B_{z}\right|$ are evaluated as a function of height for two sub-regions. These two regions (indicated by index 1 and 2) have the following solar coordinates: in subregion $1, x$ ranges in the interval: $\left[-30^{\prime \prime}, 10^{\prime \prime}\right]$ and $y$ between: $\left[-900^{\prime \prime},-875^{\prime \prime}\right]$; and in subregion $2, x$ is in the range $\left[10^{\prime \prime}, 50^{\prime \prime}\right]$ and $y$ in the interval $\left[-920^{\prime \prime},-880^{\prime \prime}\right]$. These two subregions correspond to a locally closed (1) and open (2) magnetic field region.

This is illustrated in Fig. 5, in which the square root (assumed to be representative of the electron density) of the radiance of Si I, respectively of O VI, are shown on the left, together with $\left|B_{z}\right|$ (at 14 Gauss) at $1.5 \mathrm{Mm}$, respectively $4.5 \mathrm{Mm}$, on the right. The two-dimensional plots of $B_{z}$ are obtained by extrapolation to $1.5 \mathrm{Mm}$ and $4.5 \mathrm{Mm}$. The scale of the colour coding of $B_{z}$ is given on the top of each panel. The black solid lines in the right frames show the superposed contours of the respective line radiance, which contain the regions with strong magnetic field of negative polarity (indicated in red colour). The sub-region 1 and sub-region 2 are indicated by the black rectangles on the right and white in the left frames, in which for comparison also green contour lines of strong magnetic fields are displayed. They surround regions of strong radiance in both lines.

Generally speaking, high radiance and strong field correlate well, a result which is shown in the right panels of Fig. 4, where the linear correlation plots indicate by the high correlation coefficients this close relationship. Similar results were already established in previous analyses by Tu et al. (2005a) and Tu et al. (2005b) for funnels in a CH and a quiet-Sun region. This good correlation is also obvious by inspection of the radiance isocontours superposed on the magnetic field at $1.5 \mathrm{Mm}$ in the upper right panel of Fig. 5, respectively at $4.5 \mathrm{Mm}$ in the lower right panel of Fig. 5. Similar results are obtained, and thus similar conclusions can be made, for all the other lines contained in Table 2. These results are not presented here because of the 

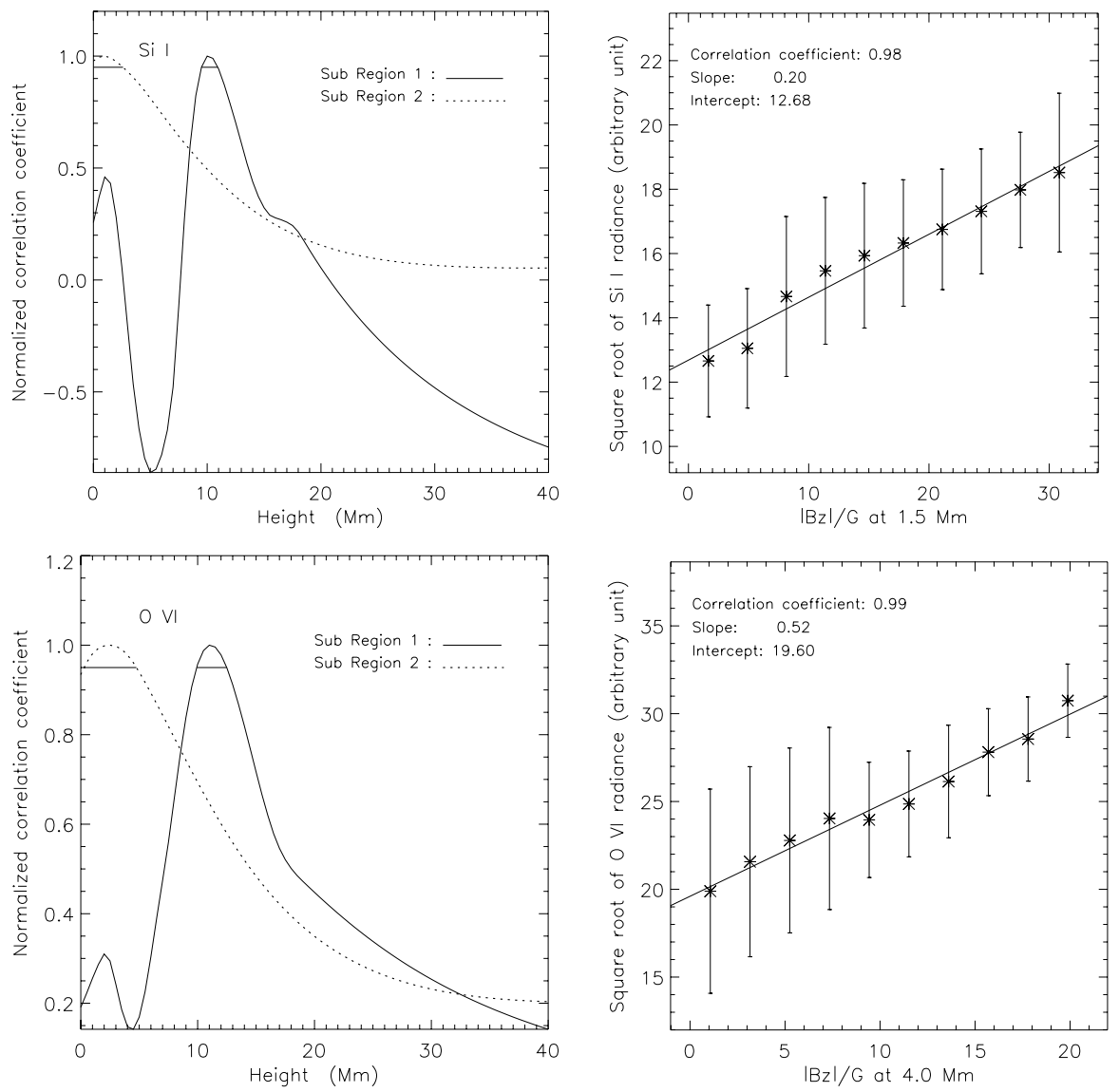

Fig. 4. Left: the height profile of the correlation coefficient between the observed line radiance and $\left|B_{z}\right|$ in two sub-regions, for Si I (top), respectively, O VI (bottom). The correlation heights in sub-region 1 and sub-region 2 (and in parentheses the height range within which the coefficient drops by $5 \%$ ) are $11 \mathrm{Mm}(9.9-12.5)$ and $2.5 \mathrm{Mm}(0.28,4.76)$ for $\mathrm{Si}$ I, respectively, $10 \mathrm{Mm}(9.50-10.93)$ and $1 \mathrm{Mm}(-0.83,2.60)$ for O VI. The correlation coefficients for Si I and $\mathrm{O}$ VI, are normalized by 0.11 and 0.40 in sub-region 1 , and by 0.51 and 0.49 in subregion 2 , indicated by a solid and dotted line, respectively. Right: dependence of the square root of the radiance of Si I (top) and O VI (bottom) on $\left|B_{z}\right|$ at $1.5 \mathrm{Mm}$, respectively at $4.0 \mathrm{Mm}$, corresponding to the average correlation height in the whole region. The asterisks show the averages of the square root of the radiance (ordinate, $y$ ) in each bin as a function of $\left|B_{z}\right|$ (abscissa, $x$ ). The uncertainties give the corresponding standard deviations of the averages. The solid lines show linear fits to the averages. From a linear correlation analysis we get $y=a+b x$, with $a=12.68 \pm 0.24$, and $b=0.20 \pm 0.01$ for Si I, and $a=19.50 \pm 0.34$, and $b=0.56 \pm 0.03$ for O VI. lack of space. However, the correlation coefficients and heights for the two subregions are presented for each line in Table 2. The heights are typically $3 \mathrm{Mm}$ for subregion 2 and $11 \mathrm{Mm}$ for subregion 1 . There are two excursions with large values, namely for S IV at $30 \mathrm{Mm}$ and Si IV at $40 \mathrm{Mm}$, large heights for which we do not have a plausible explanation other than that too low a radiance in subregion 1 resulted in an unreliable determination of the height in this tenuous open-field region.

In the top and bottom left panels of Fig. 4, the correlation heights (defined by the normalized correlation coefficient) are shown for subregion 1 (continuous line) and subregion 2 (dotted line). Their values are (and in parentheses the height range within which the coefficient drops by 5\%) are $11 \mathrm{Mm}(9.9-12.5)$ and $2.5 \mathrm{Mm}$ (0.28-4.76), respectively for Si I and O VI. The correlation coefficients are normalized by their maxima, i.e. by 0.11 and 0.40 in subregion 1 and by 0.51 and 0.49 in subregion 2 . Apparently, in subregion 1 corresponding to an open-field region with low radiance, the peaks are comparatively sharp, but surprisingly there are two peaks, indicating two heights of high local correlation. For both lines the maximum is at about $11 \mathrm{Mm}$, where one may define the top of the TR. The radiation is emitted at much greater heights than in subregion 2, where it mainly seems to come from altitudes between 2 and $4 \mathrm{Mm}$. The corresponding magnetic field structures are the smaller loops of the low magnetic carpet as illustrated in Fig. 2.

The second low peak in subregion 1 also corresponds to this lower part of the TR. It seems as if the bottom part of the openfield region is covered by small (a few $\mathrm{Mm}$ ) loops (which would not show up at the scale of Fig. 2), which have the same emission height than in the overall closed subregion 2 . The TR is stretched out to greater heights in the open corona, but remnants of the low TR in the closed corona in form of small loops are still visible. The correlation heights inferred here are in agreement with the values obtained before in a $\mathrm{CH}$ by Tu et al. (2005a) and a quiet-Sun region by Tu et al. (2005b).

Concerning the empirical correlation between the square root of the radiance (considered to be equivalent to the electron density) and the magnetic field strength, as shown in the upper and lower right frame of Fig. 4, the explanation may be the same that was given in Tu et al. (2005a) and Tu et al. (2005c). Magnetoconvection, transporting plasma trapped in loops and their associated magnetic flux together to the larger-scale flux tubes, may there reconnect and lead to a simultaneous accumulation and compression of the plasma and the frozen-in magnetic flux, to the overall effect that a close correlation is built up between plasma density and magnetic field magnitude.

The ionization temperatures (which are about equal to the line formation temperatures) and the correlation heights of the 12 emission lines observed in the two different subregions are shown in Fig. 6. Those lines having peak count rates per spatial pixel of less than twelve are not considered here. Therefore, the C IV line was not included in that plot. There is striking dichotomy between the data points of subregion 2 (black dots) and subregion 1 (open triangles), corresponding to small loops of the closed carpet, respectively to open field lines of magnetic flux forming the $\mathrm{CH}$. There are a few outlying points in both data sets, however on average the two populations are well separated. In both groups of data points, a rapid radial increase of the temperature with height is obvious. The corresponding gradients are steep and yield a jump in temperature by roughly two orders of magnitude in kelvin, over a small height interval of only about two megameters.

It is interesting to compare this with an earlier attempt by Marsch et al. (2000) to determine the hydrogen temperature 

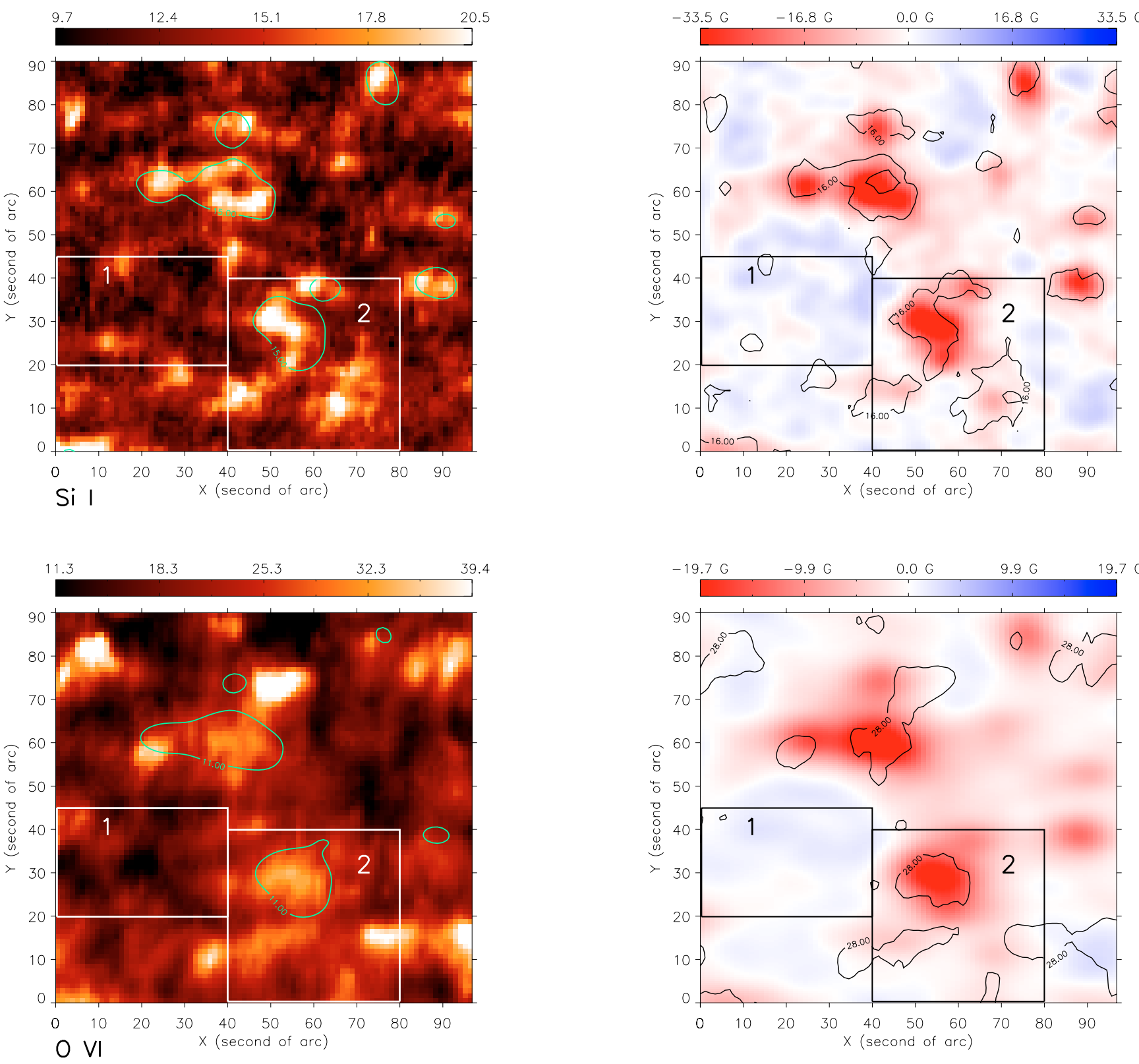

Fig. 5. Left: square root of the radiance of Si I (top) with the isocontours (in green colour) of $\left|B_{z}\right|$ at 14 Gauss and a height of $1.5 \mathrm{Mm}$, respectively of $\mathrm{O}$ VI (bottom) with isocontours of $\left|B_{z}\right|$ at 11 Gauss and a height of $4.5 \mathrm{Mm}$. The sub-region 1 and sub-region 2 are indicated by the white rectangles. Right: two-dimensional plot of $B_{z}$ extrapolated to $h=1.5 \mathrm{Mm}$ (top), respectively $h=4.5 \mathrm{Mm}$ (bottom). The scale of $B_{z}$ is given by the colour coding on the top of each panel. The black solid lines show contours of the radiance, which contain the regions with strong radiance for the Si I line (top), respectively O VI line (bottom). Sub-region 1 and sub-region 2 are indicated by the black rectangles.

gradient in a north polar region of the Sun from lines of the Lyman series of hydrogen. They found an empirical temperature jump from about 1 to $2 \times 10^{5} \mathrm{~K}$ within a height range from about 14 to $18 \mathrm{Mm}$. This course would indicate a substantial flattening with altitude of the empirical temperature gradient we found here above the top of the TR at about $15 \mathrm{Mm}$.

Figure 6 clearly illustrates the different extent of the TR, which was defined by the correlation heights (emission locations) of a selected set of lines being emitted at known formation (or electron) temperatures, for two distinct parts of a $\mathrm{CH}$, low-lying loops of the magnetic carpet and funnel-like open flux tubes reaching the higher corona. That the TR is much more extended in a $\mathrm{CH}$ has been known for a long time from off-limb observations, for example in the early TR-camera results by Bonnet \& Foing (1984) obtained from rocket flights.

Here we just gave estimates of the average heights of the line-emission sources, but we did not discuss the physical process in the source regions. For our data analysis, we did not make any assumptions, for example relating to the heating rates in loops. Schrijver et al. (2004) found that the total heating flux density in a coronal loop should be proportional to the base magnetic field strength at the loop footpoint, in order to get a better matching between model results and coronal ultraviolet images. With respect to our present results, we found that the total heating rate, while being assumed as proportional to the electron density, could indeed be in proportion to the field strength. Yet, 


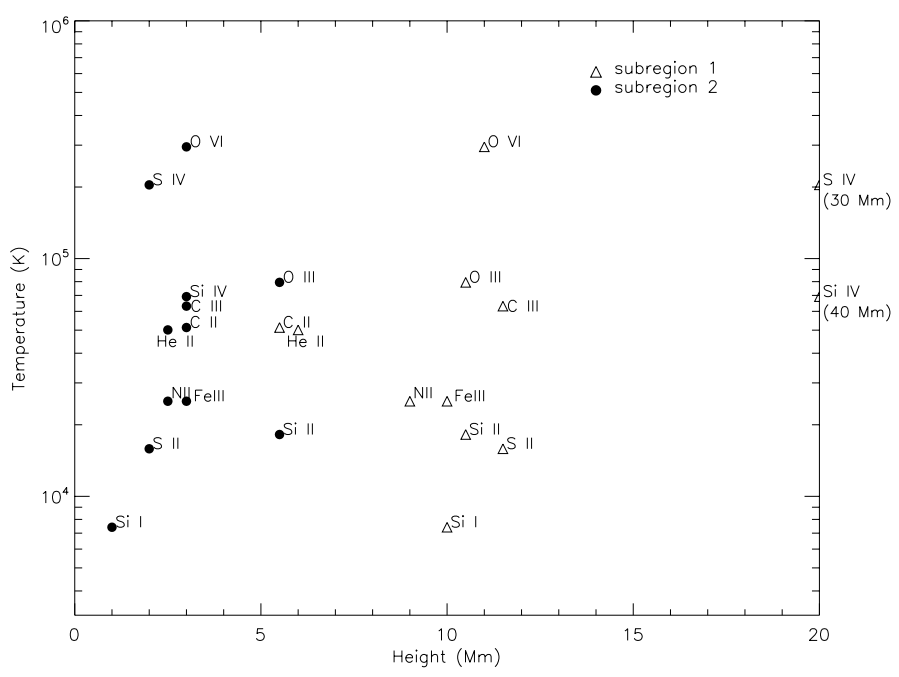

Fig. 6. Ionization temperatures and the correlation heights of 12 selected emission lines in two different sub-regions of a polar coronal hole. The triangle symbol indicates the data from sub-region 1 (open funnels), while the filled circle symbol the data from sub-region 2 (closed loops). Those lines with the peak count rates per spatial pixel being less than 12 are not considered here.

since the abundance of any single ion species is very small, its line radiation loss may be neglected in comparison with the total heating rate required to sustain the corona. Therefore, diagnosing coronal thermodynamics on the basis of a few lines is not advisable.

Our basic understanding here is that the horizontal structures in the line radiance maps are controlled by the vertical field $B_{z}$, The reason why we used the $B_{z}$ component rather than $B$ module in the correlation calculations is that the brightest transition region emissions are concentrated in the network in loop footpoints where $B_{z}$ is strongest (see Tu et al. 2005a,b).

Figure 6 shows that the ions with different ionization temperatures are located at nearly the same height, if we accept an error bar of about $1 \mathrm{Mm}$ in height. The present results support previous limb observations indicating a very steep temperature gradient in the transition region. The new finding here is that this steep temperature gradient occurs at a different average height for the closed-field and open-field region. This result means that the transition region is located at different heights in the quiet sun and the coronal hole, consistent with earlier results obtained by Tu et al. (2005a,b).

\section{Conclusions}

We analysed the coronal-hole radiance pattern in many different TR lines in close association with the coronal magnetic field. In particular, two selected sub-regions of a polar $\mathrm{CH}$ were studied, and the magnetic field correlation heights of the emission were determined for different ions. Thus we could shed new light on the complex structure of the TR at the bottom of a polar $\mathrm{CH}$. Twelve lines were used to achieve a continuous temperature coverage. Their emission heights differ substantially between the dark sub-region 1 (open) and bright sub-region 2 (closed), but reveal less variations between each other than we expected for the temperature stratification with height in a one-dimensional uniform atmosphere. In particular, regions with strong emission (multiple small closed loops) are found to be located at low heights (around 2-3 Mm), whereas the weak emission (on locally open, i.e. far reaching fields) appears to originate at greater heights $(9-12 \mathrm{Mm})$. These results indicate the coexistence of ions with different formation temperatures at about the same height in TR loops, and similarly in open fields. However, the magnetically open part of the TR in this polar coronal hole is much more extended than the closed loops, and the open TR appears to stretch out in altitude beyond $10 \mathrm{Mm}$.

Acknowledgements. The SUMER project is financially supported by DLR, CNES, NASA and the ESA PRODEX programme (Swiss contribution). SUMER is part of SOHO, the Solar and Heliospheric Observatory of ESA and NASA. We thank the NSO/Kitt Peak observatory for the use of their magnetic field data. The work of Chuanyi Tu, J.-S. He and G.-Q. Zhou was supported by the National Natural Science Foundation of China under contracts 40574078, 40336053, and 40436015. They are also supported by the Beijing Education project under XK/000/0404.

\section{References}

Aschwanden, M. J., Poland, A. I., \& Rabin, D. M. 2001, ARA\&A, 39, 175 Aschwanden, M. J., De Pontieu, B., Schrijver, C. J., \& Title, A. 2002, Sol. Phys., 206, 99

Bonnet, R., \& Foing, B. 1984, Adv. Space Res., 4(8), 43

Brekke, P. 1998, Three-dimensional Structure of Solar Active Regions, in Second Advances in Solar Physics Euroconference, ed. C. E. Alissandrakis, \& B. Schmieder, ASP Conf. Ser., 155, 150

Harrison, R. A., Fludra, A., Pike, C. D., et al. 1997, Sol. Phys. 170, 123

Harvey, K. L., \& Harvey, J. W. 1976, Sol. Phys., 47, 233

Kjeldseth-Moe, O. 2003, The Solar Transition Region, in Dynamic Sun, ed. B. N. Dwivedi (Cambridge University Press), 196

Lemaire, P., Wilhelm, K., Curdt, W., et al. 1997, Sol. Phys., 170, 105

Marsch, E., Tu, C.-Y., \& Wilhelm, K. 2000, A\&A, 359, 381

Marsch, E., Wiegelmann, T., \& Xia, L. D. 2004, A\&A, 428, 629

Moses, D., Clette, F., Delaboudiniere, J.-P., et al. 1997, Sol. Phys., 175, 571

Seehafer, N. 1978, Sol. Phys., 58, 215

Schrijver, C. J., Sandman, A. W., Aschwanden, M. J., \& deRosa, M. L. 2004, ApJ, 615, 512, 2004

Scherrer, P. H., Bogart, R. S., Bush, R. I., et al. 1995, Sol. Phys., 162, 129

Tu, C.-Y., Zhou, C., Marsch, E., et al. 2005a, Science, 308, 519

Tu, C.-Y., Zhou, C., Marsch, E., et al. 2005b, ApJ, 624, L133

Tu, C.-Y., Zhou, C., Marsch, E., et al. 2005c, Proceedings of SW 11-SOHO 16, Connecting Sun and Heliosphere, Whistler, Canada, 12-17 June 2005, ESA SP-592, September 2005

Wiegelmann, T., \& Neukirch, T. 2002, Sol. Phys., 208, 233

Wiegelmann, T., Xia, L. D., \& Marsch, E. 2005, A\&A, 432, L1

Wilhelm, K., Lemaire, P., Curdt, W., et al. 1997, Sol. Phys., 170, 75

Wilhelm, K., Dwivedi, B. N., Marsch, E., \& Feldman, U. 2004, Space. Sci. Rev., 111, Issue 3, 415

Xia, L. D., Marsch, E., \& Curdt, W. 2003, A\&A, 399, L5 